\title{
Deliciousness of food and a proper balance in fatty acid composition as means to improve human health and regulate food intake
}

\author{
Ole G. Mouritsen
}

\begin{abstract}
Diet and lifestyle are not only related to wellbeing but also have dramatic effects on the burdens of ill health and non-communicable diseases. Drawing upon anthropological evidence for human evolution and the chemistry of the brain, as well on the physiology of taste, a brief review is provided on the effects of food composition and food flavor on health, wellbeing, and the quality of life. Our ancestors' access to the particular fatty acids of marine food sources and man's early use of the fire to heat-treat food have been prime determinants for the evolution of our relatively big brains and our craving for foodstuff characterized by sweet and umami flavors. The increasing unbalance of fatty acids in our diets appears to have severe effects on the condition of our neural system and the brain, leading to a severe increase in brain disorders and mental diseases. Similarly, a lack of deliciousness in prepared meals may be a possible reason for poor nutritional management and excess intake of sugar, salt, and fat. These changed conditions are suggested as reasons why our brain, the flavor of our food, and hence our health are under siege.
\end{abstract}

Keywords: Evolution, Diet, Diseases, Food, Cooking, Nutrition, Health, Quality of life, Deliciousness, Flavor, Human head, Brain, Fatty acids, Umami, Glutamate, Taste receptor, Taste synergy, Salt, Fat, Sugar, Palatability

\section{Review Introduction}

The consequences of malnutrition and critical unbalances in the diet with regard to fatty acids, sugar, salt, and fat are becoming more and more manifest in the Western world and are gradually also influencing the general health conditions for populations in developing countries. We are currently witnessing a rapid increase in non-communicable diseases like heart and coronary diseases, obesity, diabetes, hypertension, and cancer, as well as certain mental diseases [19].

However, according to WHO's constitution [75], the health of a population is not only a condition set by the absence of illness but also determined by the "quality of life" (QOL). QOL involves aspects of social wellbeing as

Correspondence: ogm@memphys.sdu.dk

Taste for Life-Danish Center for Taste, MEMPHYS - Center for Biomembrane Physics, Department of Physics, Chemistry, and Pharmacy, University of Southern Denmark, 55 Campusvej, Odense M DK-5230, Denmark well as maintenance of full physical and mental modalities. Many countries have a growing population of elderly citizens as well as individuals who are going through complicated hospitalization and medical treatments involving drugs as well as chemo- and radiotherapy. The QOL for these individuals is often diminished due to lack of appetite, leading to malnutrition and derived secondary diseases such as gastric malfunctioning, anorexia, aphasia, and a number of other physiological dysfunctions [44]. These conditions are often exacerbated by loneliness; more and more adults are singles who eat their meals in solitude or on the move. Furthermore, elderly people in nursing homes and hospitals tend to eat less food because the food is not tasty enough or prepared and presented under conditions that do not stimulate the appetite.

Appetite, choice and acceptance of food, and the amount of food consumed, as well as the satiety and satisfaction after a meal are key factors that are intimately 
linked to the sensory perception of food [67]. Food flavor, i.e., taste, smell, mouthfeel, and chemesthetic sensation, all have influence on the amount of food taken in and the degree of satiety. The palatability of the food is therefore an important factor. Still, official dietary recommendations and guidelines [5] seldom take food palatability into account [43]. A well-known example is the recommendation of $600 \mathrm{~g}$ vegetables and greens per day which most people have problems with, presumably because of lack of palatability and taste of many vegetables. As a consequence, people eat considerably less vegetables or flavor their vegetable dishes with large amounts of salt, fat, and sugar in order to make them palatable.

In general, domestic or industrially produced meals are often made palatable by adding salt, sugar, or fat. The less palatable the dishes, the more of these additives are used. In the case of salt, epidemiological studies have indicated that the global increase in cardiovascular diseases and hypertension [3, 68] may be caused by increased intake of salt [24] although recent studies have shown that the situation is more complex [53]. Similarly, an increased consumption of sugar is believed to be a major cause of diabetes, obesity, and overweight [56]. The jury is still out with regard to fat and lifestyle diseases [34, 35], in particular regarding the role of saturated fat and cholesterol, although it is now documented that trans-fatty acids constitute a risk factor [69] and that an unbalance between the intake of omega- 3 and omega- 6 fatty acids is the cause of several neural and mental diseases $[26,46,57,66]$.

The gross imbalance in the diet's contents of omega- 3 and omega- 6 fatty acids is particularly worrisome and seems to some extent to be overlooked by the health authorities. Olesen et al. [54] have estimated the total cost in Europe in 2010 for treating brain disorders to be $€ 798$ billion, of which direct health care cost is $37 \%$, direct non-medical cost is $23 \%$, and indirect cost is $40 \%$. Concerned researchers have pointed out that the rapid growth in mental diseases, not least in young subjects, is an indication that the human brain is under siege [14]. Whereas our early ancestors, who all lived in coastal areas, enjoyed a marine-dominated diet with an omega$3 /$ omega- 6 ratio close to one, this ratio has in many Western countries become much larger than one. We eat generally far more omega- 6 than omega-3. There are many reasons for this shift. One is the rapid growth in production of cheap plant oils during the second half of the twentieth century; another is the stagnation in fisheries and limitations in the growth of aquaculture due to environmental considerations.

The unbalance in our food with respect to the contents of fat, sugar, and salt is likely only to become exacerbated unless we find a way to produce delicious and nutritious food with less sugar, salt, and omega- 6 fat.
In the present topical review, I will address these issues in a framework of evolution and taste, i.e., the flavor of food. First, I will describe the current picture of human evolution along with the discovery of cooking and man's use of fire. I will put this in perspective by referring to the evolution of the human brain and our basic sensory modalities and what constraints these physiological conditions put on our diet, not least in relation to the omnivore's dilemma [58]. Next, I will describe why I think the human brain and the flavor of our food is under siege. Finally, I will in the conclusion propose some possible solutions to the problem.

\section{Homo, the fire, cooking, and soft nutritious food}

Marks on bones indicate that our ancestors, the early hominins, started to eat meat from land animals 2.6 million years ago [77]. It has not been easy to chew and digest raw meat. It has recently been argued that the hominins used fire and heat to prepare food 1.9 million years ago which changed the game altogether [7, 77]. Homo is unique in eating a diet that is rich in cooked and non-thermally processed food [55], and this is a universal trait across all cultures and continents.

Heat treatment of meat and plant material leads to a substantial change in the structure and nutritional value of the foodstuff. Even though the early hominins are likely to have cut, torn, and mashed the raw foodstuff, cooked food is much softer to chew than tough raw meat, plants, hard seeds, and unripe fruit.

Heat treatment leads to gelatinization of starchy carbohydrates and denaturation of proteins, easier accessible nutrients, more energy (more calories), lowered infectional danger, prolonged non-perishability, and above all, less chewing and with less force. Heat treatment also leads to the release of volatile substances, and individuals who preferred cooked foods adapted to these substances as attractive taste and aroma compounds. Heat treatment made the food safer to eat, and humans may have become more accustomed than other primates to the carcinogens that can be formed in food in the course of heating it. At the same time, we have become less resistant to the bacteria that often are present in raw meat. During evolution, though, the advantages of having ready access to more calories have outweighed the disadvantages of the loss of vitamins and the possible formation of dangerous substances in cooked food.

In an evolutionary context, it is especially important that heating made the food more digestible and resulted in more readily available nutritional substances and, hence, a greater caloric intake. As a consequence, humans needed much less time to eat and to digest their food than other animals. This gave us some unique and distinct evolutionary advantages over those species that had to devote 8 to $10 \mathrm{~h}$ a day to obtain their food supply 
and possibly an equal amount of time for chewing it. For example, chimpanzees and other large apes currently spend more than $6 \mathrm{~h}$ chewing every day [78].

"Chewing" for a long time to make the nutrients accessible from raw foodstuff is precisely what modern contenders for the raw food movement have to do in order to get sufficient nutrition, but now helped along by modern food processors. Otherwise they would also be forced to chew most of the day. We no longer have teeth that are designed to chew raw meat and plants, and typically 50-70 movements of the jaws are needed to comminute a dry piece of meat [38]. Experiments have furthermore shown that we, as a species, could not survive on a diet of raw food, as it provides too little energy, leads to weight loss, and most importantly, reduces reproductive capacity [77].

Comparisons between human anatomy and that of other primates show that we have smaller teeth, weaker jaws, and a small mouth [77]. Another characteristic that distinguishes us from the other primates is a smaller stomach and shorter intestine in relation to body size. This seems to be an indication that our digestive system has adapted to prepared food, consumed in smaller pieces, which can be converted in less time into more nutrients and more energy. Consequently, as the digestive system required less fuel to carry out its work, more calories were available for the muscles and the brain. Evolutionary biologists regard the period 1.8-1.9 million years ago as a time of transition from ape species (Australopithecines) to the hominins Homo erectus, who had a smaller brain and a lower brow than modern humans. It is presumed that an important driving force for this evolution was that, during this transition period, the ancestors of Homo erectus, the habilines, became huntergatherers and changed from herbivores into omnivores. Homo sapiens are thought to date back about 200,000 years [77].

Wrangham is of the opinion that the culinary arts have been instrumental in the development of social structures and the division of labor according to gender [77]. Men undertook the arduous, risky task of hunting, while women spent their days gathering fruits, plants, and roots as well as kept the fire going for the men to return with their catch, both sexes possibly contributing equally to the pool of calories for the family [17]. The inevitable socializing associated with the eating of a warm meal was, at the same time, a stabilizing factor that fostered the creation of family and societal ties, according to Wrangham.

Another consequence of the diminished need to chew the food is according to Lieberman $[38,84]$ the particular shape of the human head (cf. Fig. 1). It is a characteristic trait of modern humans that we have a flat face, no snout, and relatively small teeth. Apes have longer faces and more protruding and stronger jaws, which reflects how the pressure is distributed in the cranium when they chew their tough food. Lieberman hypothesizes that changes in the diet, in particular the arrival of soft food via use of the fire, have led to changes in the shape of the human face.

Heads are a 500-million-year-old invention, and the human head has been subject to a selection pressure determined by not only by the way we walk and the way we run [39] but also the way we eat. The head, and in particular the oral cavity, teeth, and pharynx, is of major importance for the way we taste, masticate, and swallow the food. The texture of the food, in particular its softness, hence becomes a determinant of the evolution of the human head.

Soft food requires less and less forceful chewing, and Lieberman has estimated that the modern human being needs $30-50 \%$ less power in the jaw muscles compared to that of the stone-age man. It has indeed been found that the expression of myosin, the molecular motor that drives the muscular motion of the jaws, has been downregulated during human evolution [40]. The form of the modern human head, with a small outer nose, a rather short inner nasal cavity, and a small face, implies that our pharynx is relatively short. This head design is, according to Lieberman, essential for the way we breathe and our ability to moisten the inhaled air and dehydrate the exhaled air, thereby securing better thermoregulation and great effectiveness at exhausting runs [39].

The energy in heat-treated plant material derives predominantly from hydrolyzed starch that is degraded into digestible sugars by amylase, an enzyme that is found both in saliva and is secreted from the pancreas to the intestines. Although we can live without starch, $50 \%$ of the calories in the diet of a modern man come from starch.

As a special bonus from the evolution of cooking and the culinary arts, the food not only became more nutritious, it also became more tasteful. Or we should rather say that humans evolved senses that could steer us towards food that is rich in calories and rich in proteins. The primary tastes sweet and umami do exactly that, respectively [51]. Cooking and fermenting meat and vegetables release large amounts of sugars, free glutamate, and free nucleotides and hence lead to food and meals enriched in sweetness and umami.

It is a universal trait among humans, independent of culture, to crave foodstuff that taste sweet or umami, whereas our appreciation of bitter is a consequence of adaption, tradition, and culture, because bitter is often a signal of poisonous food. The two remaining basic tastes, sour and salty, are likely to be important for us to steer away from unripe fruit and to acquire a proper $\mathrm{pH}$ and electrolyte balance in our bodies [60]. 


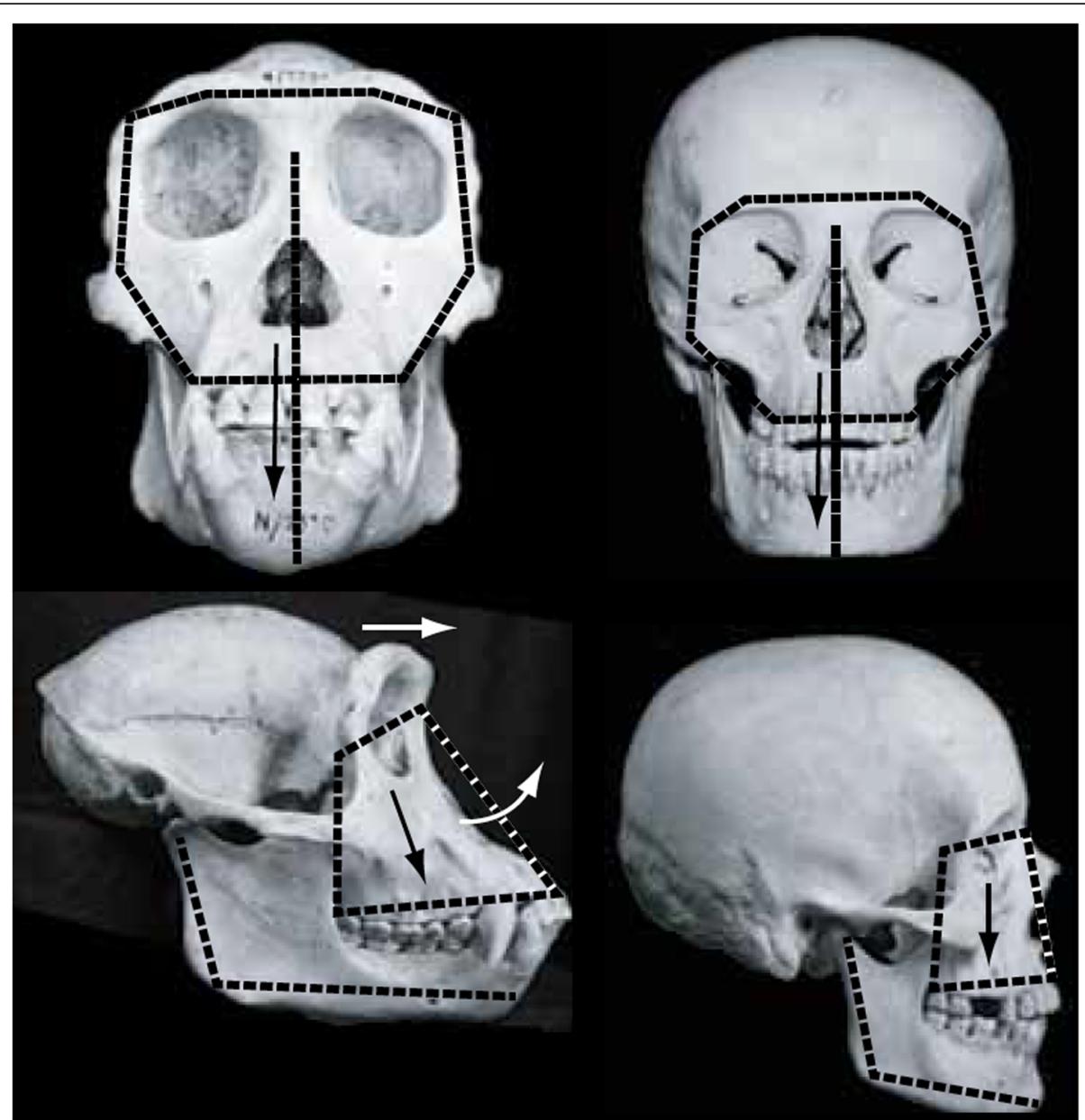

Fig. 1 Front and side views of adult chimpanzee (left) and human (right) skulls exhibiting the differences in facial shape as a consequence of the different modes of chewing the food. Courtesy of Dr. Daniel Lieberman

Hence, it appears that good nutrition and especially umami most probably go hand in hand with the evolution of humankind over the past two million years or so, and our preference for delicious food is encoded in our sensory system over millions of years. In particular, the umami taste sends a clear signal that the food is nourishing and, consequently, important for survival. There is some indication that the diet of modern humans is not properly adjusted with respect to umami, and we shall later return on how this may influence nutritional status and public health.

The extra energy that has been made available after man became a cook has not only made it possible to spend less time in eating and digesting the food and more energy for physical activities, but it has also made it possible to create the conditions for the evolution of a large brain that for an adult consumes more that $20 \%$ of the total energy at the disposal of the body as a whole [13]. Paradoxically, and as a consequence of the omnivore's dilemma, we spend a fair amount of this energy pondering about what we actually should eat and what we dare to eat [58]. A further development that served to conserve energy was the evolution of a significant layer of body fat [12], a unique feature of hominins compared to the greater apes.

However, in addition to lots of energy, there is yet another requirement for the evolution of the human brain; the brain could not have evolved without access to some special building materials, in particular elements like iodine, iron, zinc, copper, and selenium, and highly specialized essential fatty acids, the highly unsaturated omega- 6 and omega- 3 fatty acids [14]. In the context of the nutritional status of modern humans, it is the diminished amount in our diet of two special omega-3 fatty acids, DHA (docosahexaenoic acid) and EPA (eicosapentaenoic acid), that is a growing global threat to the health of the population.

\section{Evolution of the human brain}

Neural systems and the brain share a common trait among all organisms that have neural systems. The 
neural membranes contain large amounts of highly unsaturated fatty acids [15]. Of the omega- 6 variety, it is particularly arachidonic acid (20:4) and adrenic acid (22:4), and of the omega-3 variety, it is DHA (22:6) and EPA (22:5). More striking is that the total amount of all omega- 6 fatty acids is close to the total amount of all omega-3 fatty acids in the brain, i.e., the ratio omega-3/ omega- 6 is close to 1 (cf. Fig. 2). It is striking that this ratio in marine foodstuff such as fish, shellfish, and marine algae is of the same order of magnitude.

One of the characteristics of humans as a species is that our brains are large in proportion to our body mass. Roughly, we have a brain whose mass is about ten times as large as it would be if it followed the expected logarithmic dependence of brain/body weight ratio as a function of body weight (cf. Fig. 3). It is now generally acknowledged that Нomo erectus did not evolve on dry, warm grasslands but in the damp, warm regions that formed the border between land and water [10, 15].

Michael Crawford has pointed out that the allimportant sources of DHA and EPA can only be found in sufficient quantities in littoral areas, where fish, shellfish, and algae are abundant [11]. These fatty acids are critical for the formation of a complex nervous system and a large brain. The access to these fatty acids is therefore considered to be a determining factor in the evolution of modern humans that our ancestors, during the period from about $1,000,000$ years ago until the appearance of the first modern Homo sapiens about 100,000-200,000 years ago, had a diet that consisted of fish and shellfish.
However, since seafood also contains certain micronutrients that are known to be important for brain development [14], it may be that DHA and EPA are not alone the determining factors. Stephen Cunnane has studied the relationship between evolution and nutrition and drawn attention to the fact that the development of the brain is especially dependent on five micronutrients: iodine, iron, copper, zinc, and selenium [14]. Without a sufficient supply of these, the genetic potential for the evolution of a large and complex brain could not be maximized. Iodine plays a particular role because it is a prerequisite for the production, in the thyroid gland, of two important iodinated hormones, thyroxine and triiodothyronine, which regulate metabolism and control growth rate. It is Cunnane's hypothesis that hominids living far from the sea would have been unable to obtain enough iodine to evolve a brain such as that found in humans today. Although freshwater aquatic food sources would contain enough of highly unsaturated fatty acids and minerals, a shift from freshwater aquatic resources towards marine food resources would have improved the conditions for brain development due to the greater abundance of iodine in the marine environment [30].

\section{The human brain under siege}

The development of the human brain in modern humans, during the fetal stage and in the first years after birth, in fact up to age 18 although less aggressively, reflects the evolution of the human brain and the need for food supplies rich in DHA [4]. Being our most fatty

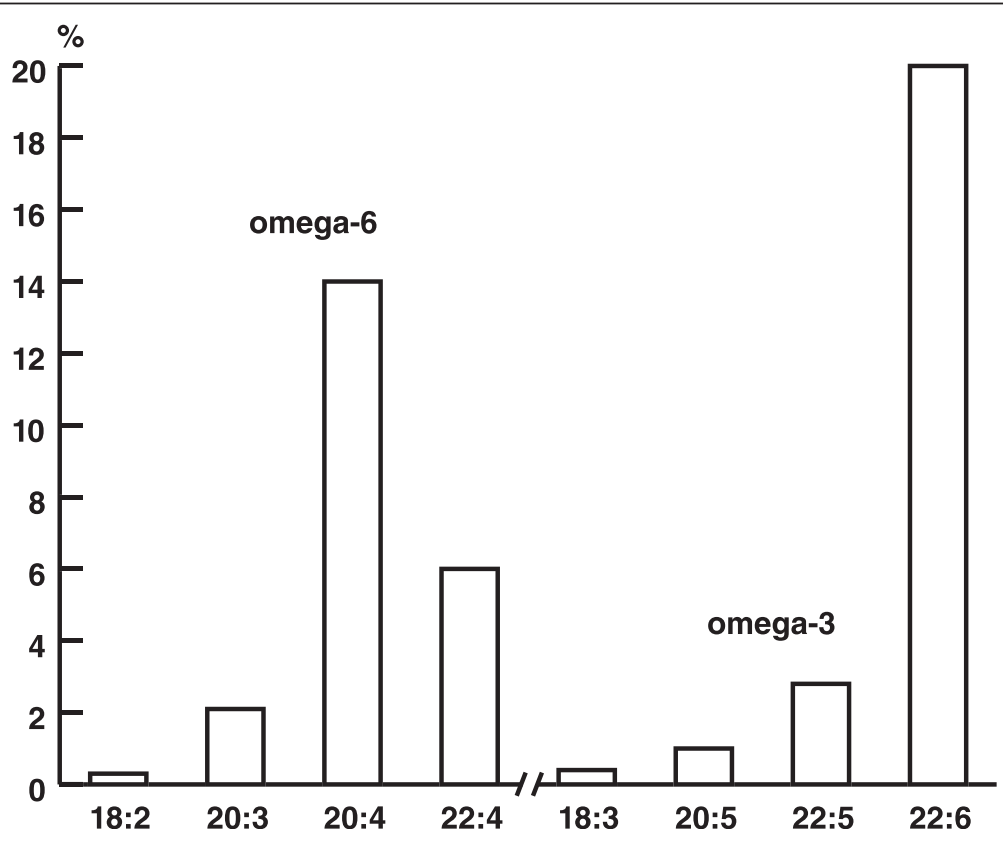

Fig. $\mathbf{2}$ Distribution of poly-unsaturated fatty acids in the motor cortex of the human brain, highlighting the omega- 6 and omega-3 fatty acids with a predominance of arachidonic acid (20:4) and docosahexaenoic acid (22:5), respectively. Courtesy of Dr. Michael A. Crawford 


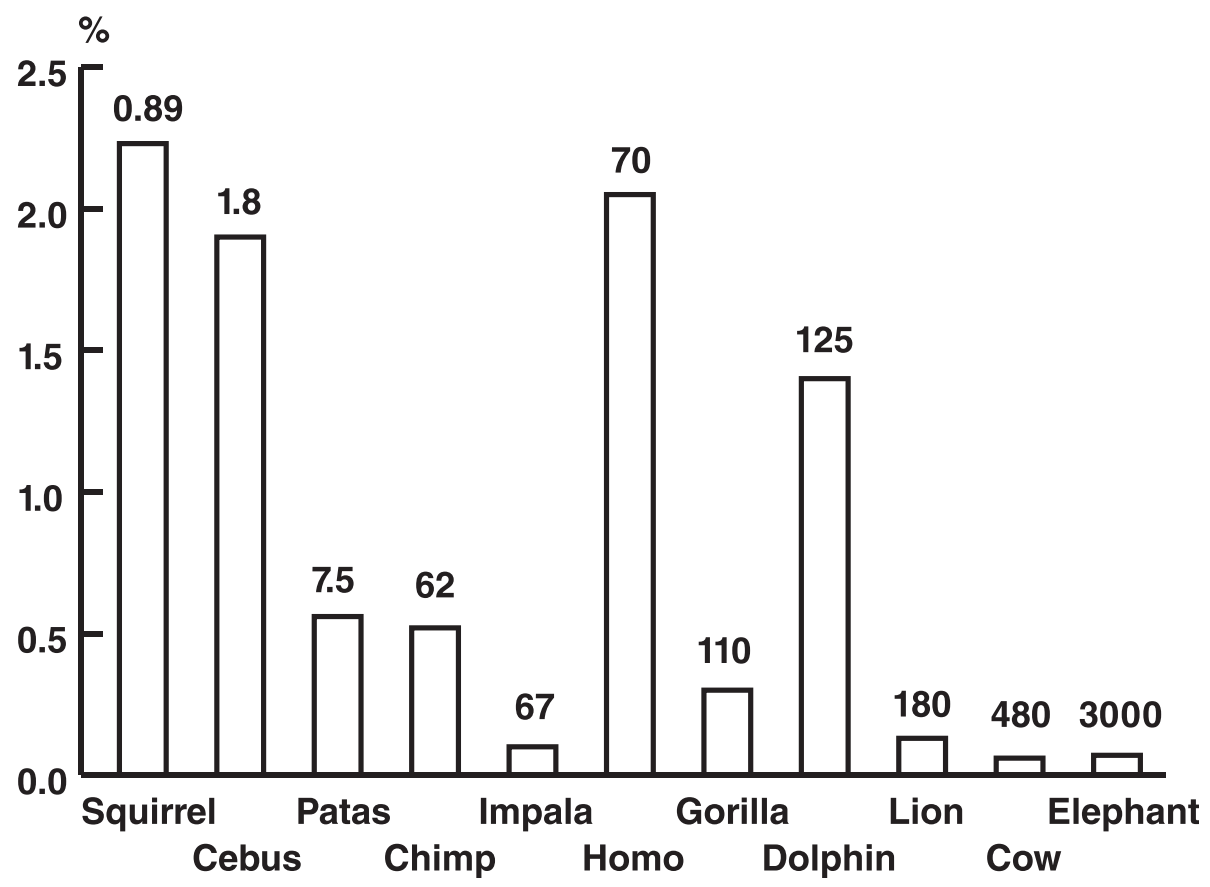

Fig. 3 Brain mass as a percentage of body mass for a series of small and large animals. The numbers above each bar denote the brain weight in kilograms. Courtesy of Dr. Michael A. Crawford

organ, the brain consists of more than $70 \%$ fat, most of this being highly unsaturated omega- 3 and omega- 6 fatty acids (cf. Fig. 2).

Omega-3 fatty acids (as well as omega-6) are essential fatty acids, and they have to be supplied by the diet because the conversion rate from lower-grade omega-3 sources, such as plant-based $\alpha$-linolenic acid, is not very efficient in humans. Fish, shellfish, and algae are a major source of these fats. For example, DHA makes up $50 \%$ of the fat content in the muscle tissue of a fatty salmon, whereas it makes up only $0.2 \%$ in beef. Some farmed fish, like tilapia and catfish, due to their feed being based on plant oils high in omega-3, have a fatty acid profile very different from wild fish, whereas salmon appears be more robust to the fatty acid composition of their feed [74].

Whereas the diet of the hominins living in coastal regions consisted of marine or freshwater foodstuff not only rich in highly unsaturated omega-3 fatty acids but also with a omega-3/omega- 6 ratio close to unity (typically in the range 0.3 to 1.8 ), the diet of modern humans has a much smaller ratio, typically in the range 0.04 to 0.15 . That is, we eat $10-20$ times too much omega- 6 on the expense of omega-3. It is important to notice that, since the total mass of food you eat is more or less constant, it is not enough only to eat more omega- 3 but also to reduce the intake of omega- 6 to restore the fatty acid balance in the diet. Too much omega- 6 will "wash-out" the omega-3 [46].
Therefore, access of DHA and EPA plays a seminal role in building up our central nervous system, especially the brain and its visual forefront, the retina of the eye. In contrast to the saturated fats ingested from animal products, DHA and EPA also prevent cardiovascular disease and cancer and help to regulate the cholesterol count in the bloodstream. In contrast, too much omega6 in the diet elevates the risk for inflammatory diseases. In particular, DHA activates over 100 different genes, lowers the risk of death after a heart attack, regulates the immune system, and improves cognitive abilities in children [10].

Many researchers have pointed out that the imbalance in modern humans' diet skewed away from omega-3 fatty acids is a major reason for the rapid growth in neural and brain-related diseases [25, 46, 54], such a bipolar diseases, depression, and a wide range of other mental disorders. This is particularly worrisome since the occurrence in young subjects is on a steep rise, reflecting the developing brain's need for omega-3 fatty acids. Research has shown that the increase in some psychiatric disorders can be attributed to an imbalance between omega- 3 and omega- 6 fatty acids in the diet [25]. The present state of knowledge in these matters is still limited and insufficient to lead to concrete recommendations that go beyond general nutritional advice. But there are indications that just as cardiovascular disease was the Achilles heel in Western societies in the twentieth century, the central nervous system-particularly the 
brain - will be the next area of vulnerability, and the human brain will become under siege.

The brain is most vulnerably when it is under development in the fetus and in young children [21, 27, 42]. DHA is a factor in the development of the nervous system and brain of the fetus and the infant, especially vision and cognitive proficiencies. While it is difficult to study the cognitive development of children, there are indications that it is positively correlated to the intake of DHA. The fetus draws heavily on the DHA supply of the expectant mother. In fact, the fetus depletes it to such an extent that children from subsequent pregnancies get less DHA from the mother. It should also be noted that mother's milk is rich in DHA. It is indisputable that DHA is vital in early childhood development. But the DHA content of mother's milk varies greatly, being dependent on the composition of the mother's food intake.

It has been found that children born to mothers, who eat fish in large quantities, have better visual and cognitive abilities. These conclusions were drawn from a study of about 15,000 pregnant women in England and their children [27]. A low intake of fish by pregnant women leads to poorer fine motor and incomplete neurological development in the children. Over and above all of this, it appears that women who eat more than the recommended intake of fish give birth to children who are more advanced cognitively. Interestingly, no adverse effects from possible methyl mercury contamination of the seafood could be detected even when the mothers had a very large intake of fish during pregnancy.

Altogether it appears that there is a severe need to pay more attention to the balance of the fatty acids in the diet of modern humans. This is not necessarily a simple issue since it remains unclear whether there is a difference in the possible effects on the neural system between supplying the essential fatty acids via whole foods or by supplementation [9].

\section{The flavor of food under siege}

The human modalities of taste sensation are likely to have been shaped and conditioned during evolution [33, 51]. It is believed that the human preference for sweet direct us to food with carbohydrates and plenty of energy and calories; saltiness indicates foods with vital ions, such as sodium and potassium; sour suggests whether a fruit or vegetable is unripe; and bitter is a warning signal of poisonous compounds. Hence, the ability to taste these four basic tastes has been subject to an evolutionary pressure. Finally, for most kind of foodstuff umami signals high contents of amino acids and proteins.
Umami may consequently be an ancient signal of nutritious food with a large and easily accessible protein content. The evolutionary advantage, encoded in the human neural system, of sensing umami is supported by recent brain imaging studies $[1,6]$ which show that umami is represented in the anterior part of the orbitofrontal cortex, a cortical region which represents information about the reward value of other primary and secondary reinforcers as well as gustatory responses [1]. It is interesting to note that the human fetus and the newborn baby are primed to savor umami. Amniotic fluid and human breast milk both contain free glutamate. In fact, mother's milk is distinctly characterized by both sweet (lactose) and umami (glutamate) flavor.

In many respects, the basic tastes sweet and salty are key regulators of flavor in modern industrially produced food. When we add that this type of food may be very fatty with an improper balance of the fatty acids, it becomes clear why the food we like to eat [60] is also the food that threaten our health and contributes to the global burden of ill health, including obesity, diabetes, hypertension, cancer, and mental diseases.

It appears that umami, in particular in the Western world, is overlooked as a powerful (and evolutionary determined) way to impart delicious taste to food and meals that are also healthy [22, 44, 51, 79, 82], although we have plenty of sources of umami in the Western cuisines. The umami taste is often associated with meaty and savory flavors. These flavors are seldom present in the raw and untreated ingredients, except for unique foodstuff like ripe tomatoes and certain seaweeds. Intensive umami flavor is mainly produced and released after processing the foodstuff, i.e., by cooking and preparation. During the time since man became a cook, he has learned to employ processes that produce umami taste, such as boiling, simmering, roasting, broiling, smoking, drying, maturing, aging, storing, conserving, and fermenting (by microorganisms or enzymes). Of all these processes, fermentation is the most powerful to create deliciousness and umami.

It is interesting to note that, in the classical culinary triangle proposed by Lévy-Strauss [37], the raw foodstuff can be changed either by cooking (temperature) into edible foodstuff or by microbiological degradation into rotten and inedible stuff. "The cooked" and "the rotten" then represent culture and nature, respectively. However, the borderline between "the cooked" (prepared) and "the rotten" is not sharp, and different cultures as well as cultures at different times have varying perceptions regarding what is considered edible and what is not [28]. As pointed out by Vilgis [73], by considering fermentation and long-time cooking as a desirably way to degrade proteins and carbohydrates in prepared food, in much the same way as spontaneous degradation in undesirable 
putrefaction, the borderland between "the cooked" and "the rotten" tends to be blurred.

Fermentation releases a range of flavorful taste compounds and aroma substances. However, a bad smell can often overpower the desire to taste the fermented foodstuff. In many cases, the fermentation processes produce a bounty of nutritional elements that are more easily digestible and provide for enhanced bioavailability in the gastro-intestinal system. At the same time, properly conducted fermentation leads to preservation and prolonged lifetime of the prepared foodstuff, e.g., via dehydration or production of alcohol and lactic acid that suppresses undesired fungal and bacterial growth as well as putrefaction.

The point is now that all the processes mentioned above for eliciting umami taste require precious time that is a commodity many people nowadays prioritize to use for other purposes than working in the kitchen. Consequently, caring for the deliciousness of food for a large part of the population is left in the hands of caterers, restaurants, and large companies who often use the easy way out only adjusting sweet, salty, and fatty tastes in mass-produced foodstuff. In this sense, the flavor of our food is under siege.

It is known [51] that umami is stimulated by free monosodium glutamate (MSG), the sodium salt of the amino acid glutamic acid, in a peculiar synergy with certain free $5^{\prime}$-ribonucleotides, specifically inosinate (inosine-5'-monophosphate, IMP) and guanylate (guanosine-5'-monophosphate, GMP), which in a synergistic fashion potentiate the umami receptors' sensitivity to glutamate $[47,83]$. Umami can be considered the essence of deliciousness, which can enhance the sensory perception of a meal and moreover limit the craving for salt, fat, and sugar.

Umami can be imparted to food by natural occurrence of umami compounds or by adding these artificially as food additives [51]. The controversial issue of adding MSG to foodstuff has recently been reviewed by Mouritsen [45] who concludes a study of the available literature by pointing out that MSG is the most carefully studied food additive and is no more dangerous than ordinary kitchen salt.

Umami may be part of the solution to provide healthier, less caloric, and more satisfying meals to the population. Control of and optimization of umami may be a key to regulate and adjust food intake to the levels that are natural for human physiological functions. Studies have shown that it is possible, by regulating umami taste, to reduce the fat content by up to $30 \%$ without compromising palatability [2]. The optimal way to achieve this is however very dependent on the type of food as well as individual preferences.
Umami can show a way to reduce salt intake up to $50 \%$ while retaining palatability [80]. A particular interesting possibility with added functionality is the use of certain seaweeds for providing umami flavor in foods [48] and hence reducing the need for adding salt. At the same time, seaweeds contribute their own salts that generally are dominated by potassium salts rather that sodium salts [49] and appropriately chosen seaweeds can serve to reduce salt in, e.g., meat products.

Umami also enters a synergistic relationship with sweet taste. MSG cannot replace sugar, but it turns out that in foodstuff with a little sweetness, addition of pure MSG or other foodstuff with a natural occurrence of free glutamate can enhance the sweetness substantially [51]. The mechanism behind this enhancement is not understood in detail but may reflect that the umami receptor dimer T1R1/T1R3 shares the component T1R3 with the receptor for sweet, T1R2/T1R3 [70]. By this type of sweetness enhancement, umami can help to suppress undesirably sour taste in some foodstuff.

Hence, paying more attention to taste and what provides deliciousness to food may be a way to cut down on salt, sugar, and fat. But there is more to it.

Glutamate is vital for the normal functioning of the digestive tract and our digestive system [61]. Glutamate has been found to stimulate saliva and immunoglobulin A secretion which have implication for immunological and nutritional status [72]. Therefore, umami stimulates appetite and is important for oral as well as overall health [64]. Glutamic acid and glutamate are effectively absorbed in the gastro-intestinal system by an active transport system located in the mucosal cells where it is metabolized and turned into energy, most of it consumed for digestion. Excess glutamate in the bloodstream is degraded in the liver. The stomach and the intestines have a developed system of nerves that to some extent function autonomously and independent of the oral taste system, but it is also connected to the brain via the vagus nerve that constitutes a gut-brain axis (cf. Fig. 4). It is now known that there are glutamate receptors throughout the gastro-intestinal system [63]. Binding of glutamate to these receptors signals, via the vagus nerve, to the brain. The brain in turn signals back to the fundic glands to release proteases, thereby providing for gastric phase regulation of protein digestion. Only glutamate, and none of the other amino acids (or glucose or sodium chloride for that matter), can activate the signaling pathway along the gut-brain axis. By coupling to this pathway, sensing of glutamate at the oral umami receptors could possibly be a signal to the body that protein-rich food is on the way. The feedback to the brain along the gut-brain axis is a signal of satiety and satisfaction [32]. In this way, umami taste sensed at the taste buds at the tongue may facilitate the body's 


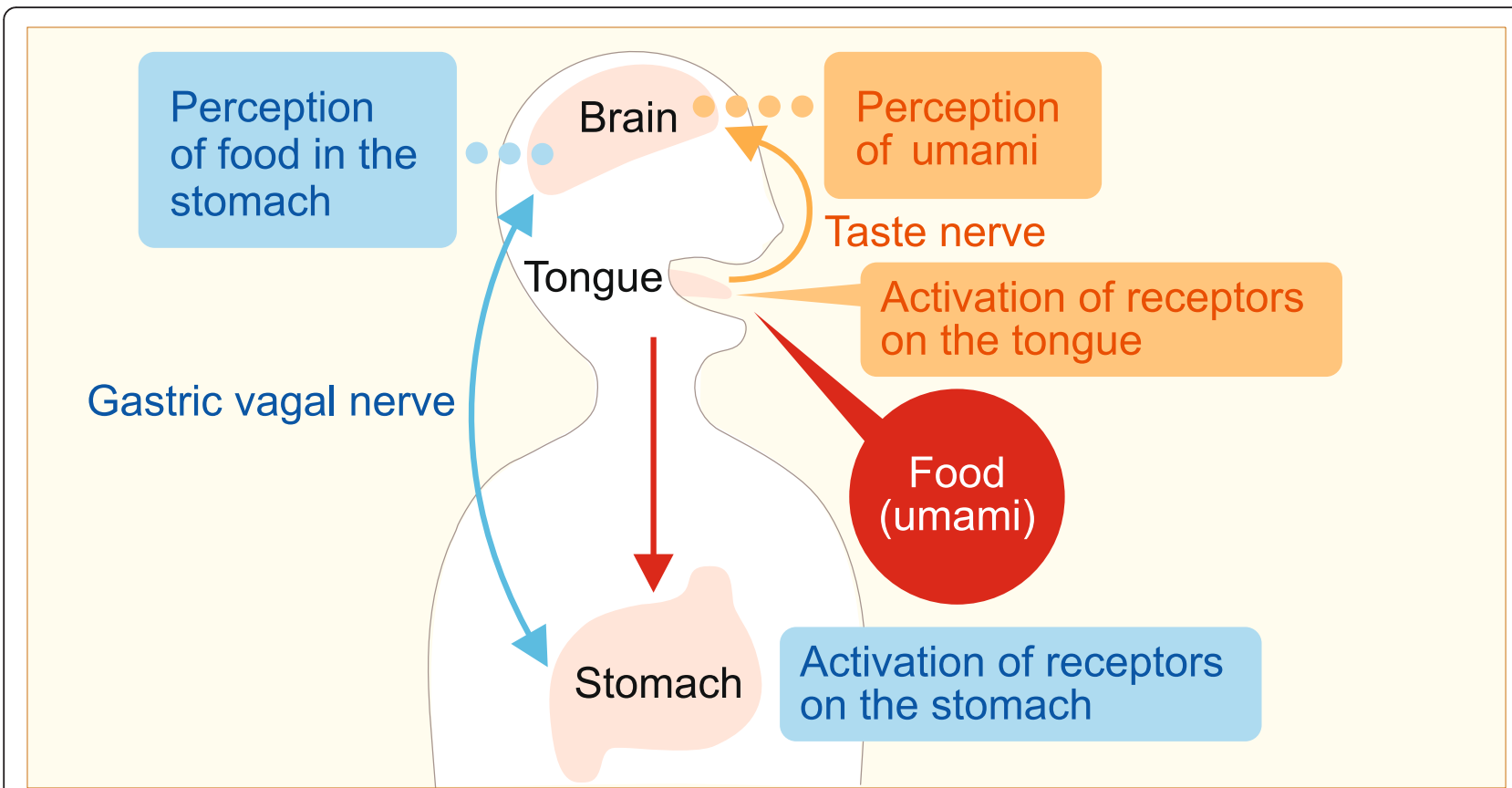

Fig. 4 Schematic illustration of the gut-brain axis highlighting the feedback loops entering the relationships between umami taste, satiety, and control of food intake. With permission from the Umami Information Center, Tokyo

alertness to be ready to digest the food and regulate appetite and food intake. The total effect of these mechanisms is a homeostatic control of energy.

Therefore, umami taste via glutamate is a key to regulate appetite and satiety. There have been some reports suggesting that glutamate may induce obesity and overweight, but recent studies indicate that this is not the case [18].

Altogether, it appears that there is a need to focus attention on what provides deliciousness to food, and umami may be part of the solution to provide tasty, healthy, and nutritious meals without excess of salt, sugar, and fat.

\section{Discussion}

I have in the present paper argued that our brain and the taste of our food are under siege. The reasons are claimed to be, respectively, a gross imbalance in the fatty acid composition of our food and a lack of attention to the food's flavor and deliciousness that control appetite, satiety, and regulation of food intake. There is now a substantial knowledge about the relationship between fatty acid composition of food and the function of our neural system [14, 57]. Recent years also have witnessed a stronger scientific interest in taste and how it relates to health and wellbeing $[50,52]$. The questions naturally arise as to which extent we are willing to and capable of using this insight to improve the conditions for the global population. This is, however, not only a matter of scientific insight but also a question of natural resources and political initiative.

The prime source of omega- 3 fatty acids and foodstuff with a proper omega-3/omega- 6 balance is the marine environment. Present knowledge suggests that the advantages of including fish in the diet outweigh the drawbacks from possible sources of contamination, in particular, if certain fish species are avoided. Hence, there is no reason to discourage North Americans and Europeans from increasing their consumption of fish and shellfish, even though there is still a lingering unanswered question concerning what the recommended weekly intake should be. Many experts are in agreement that there is too much emphasis, not least in the popular media, on the risks instead of on the actual healthful benefits. Even though we know that population groups who eat a great deal of fish are generally healthier, live longer, and have a lower incidence of cardiovascular disease, we seem to hear only the negative reports. People have a neurotic relationship with their food, and they would rather avoid foodstuffs with a low or hypothetical risk of being harmful than take their chances on ending up with diet-related diseases [36].

Recommending more fish in the diet leads however to another problem. If the recommendation is that we should eat more fish, possibly twice as much, where will the supply come from? Globally, the catch is either stagnant or decreasing and there are serious concerns about the viability of some fish stocks. It is estimated that more that half of the world's species of edible fish are 
being used to capacity, about a quarter are being overfished, and only the remainder are not fully harvested. Given that the per capita consumption is approximately constant and that the world's population is increasing, it is already apparent that there will be scarcities. It is expected that by 2030, we will have a global shortfall of up 50-80 million metric tons.

In view of the fact that farmed fish presently account for more than $40 \%$ of the total production [20] and is growing much faster than capture, one might think that a greater resort to aquaculture could help the problem. But additional production from this source will have difficulty in making up the deficits because the current output is increasing by just a few percent annually and the increase is no match for the demand created by population growth. It is interesting to note that $90 \%$ of the farmed fish originate in Asia, especially China, and the largest proportion of this supply is destined for the European Union, Japan, and the USA. Growth in fish aquaculture is severely limited by environmental concerns regarding massive pollution from fish farms. Added to this is the problem as how to produce enough feed to the farmed fish, in particular the carnivores that only tolerate a certain level of plant oils in their feed. There is yet another caveat in that farmed fish fed on plant oils obtain a fatty acid profile that can be massively different from that of wild fish, with a much larger proportion of omega- 6 fatty acids [74].

The conclusion to be drawn seems to be that without a completely different approach to the administration of the oceans' bounty, there may not be enough fish to go around.

But there may be another marine source to tap, which is in fact the original source of the precious highly unsaturated fatty acids: the marine algae [49]. It is the algae, both microalgae and the large macroalgae, i.e., the marine seaweeds that can synthesize the long-chain highly unsaturated fatty acids that, via the food web, accumulate in fish and shellfish. To put this in perspective, it should be noted that the algal kingdom is by far the largest biological kingdom on the planet, being responsible for production of the majority of organic matter (and hence fixation of carbon dioxide) and the majority of the oxygen in the Earth's atmosphere.

Eating more marine algae could be part of the solution to the problem of finding additional sustainable ways of providing food for a hungry planet. The world's total industrial production of seaweed amounted to 19 mio tons in 2010 [20]. The farming of seaweeds is on a rapid increase with growth rates between 7-8 \% annually, with almost $80 \%$ of the production taking place in China, Indonesia, and the Philippines. In 2010, the wild harvest of seaweeds amounted to only $4.5 \%$ of the total production [20].
Whereas seaweeds are an integral part of the diet in many Asian countries, in the Western world, there are only small pockets of traditional uses of seaweeds as food $[49,59]$, although seaweed extracts are used extensively as additives in the food industry in the form of hydrogels and thickeners. Further utilization of wild and farmed seaweed in the Western world will require research and development of new methods and technologies for cultivation, harvesting, and processing of the seaweed varieties with which we are already familiar, as well as with some entirely new ones. Most of all, it will also necessitate insightful planning that respects the ecological cycles of the oceans and the Earth and has to take into account the needs of the populations that live along the coastlines and depend on the resources of the sea. Finally, a change of the mindset of the consumers needs to be worked on in order to open up for the use of seaweed products in the common diet $[44,49,62]$.

It is difficult to determine conclusively whether seaweeds were also incorporated into the diet of our distant ancestors [49]. Studies of fossil finds indicate that the enamel surface of the teeth of early hominids shows evidence of wear that is characteristic of eating food containing a certain content of silica particles, which are typically found in wetland plants. In addition, analysis of the minerals found in fossilized bones has shown that the proportion of strontium to calcium is so low as to suggest that these individuals were not very high up in the food chain and, consequently, were probably herbivores. So, it is reasonable to suppose that seaweeds, given their prevalence in coastal areas, also played a role in the early hominid diet.

It is the seaweeds' contents of high amounts of minerals, proteins and essential amino acids, trace elements, vitamins, iodine, low-calorie dietary fibers, and polyunsaturated fatty acids, which lie behind their many nutritional qualities as human foodstuff. This is well recognized in, e.g., China, Korea, Polynesia, and particularly in Japan where seaweeds are an essential part of the classical cuisine and highly regarded for nutritional value, flavor, and texture. Moreover, seaweeds contain a range of antioxidants and other bioactive compounds that together with the dietary fibers and the perfect balance between omega- 3 and omega- 6 fatty acids create a role for seaweeds in the amelioration of certain risk factors associated with cardiovascular diseases [8].

Returning to the question of the importance of flavor for health and QOL, we revert to deliciousness and umami. The preference for high-fat food has been claimed to be a universal human trait [16]. Fat has aroma and contributes to the mouthfeel and texture of food, which are considered desirable. However, lessons from the classical, strictly vegan, Japanese temple cuisine, shöjin ryōri ("The enlightened kitchen")

[23] 
demonstrate that it is possible to produce healthy and delicious food virtually without using fats and oils. The trick discovered by the Buddhist monks is to enhance umami by appropriate choices of combinations of ingredients. It is interesting that until recently obesity was not a major problem in Japan. A possible reason may be that the traditional Japanese diet focuses on umami. The changes in the Japanese food culture along the lines of the globalized and industrialized Western cuisine is probably the reason why diet-related diseases are now also on the rise in Japan.

Elderly individuals as well as people who are subject to intensive hospitalization and chemo- and radio therapeutic treatments may suffer from permanent or transient impairment of taste and smell faculties which leads to loss of appetite, resistance to eating, malnutrition, and in some cases anorexia [76]. All these conditions tend to lower QOL. Increasing umami in the meals may be part of a solution to their problems. A simple way to do this is by reduction and concentration of flavor compounds in boiling water and sauces. At the same time, increasing umami will lower the need for salt and sugar in the food. Glutamate stimulates appetite and enhances saliva and immunoglobulin A secretion that leads to a smoothening of mastication and swallowing [72]. Immunoglobulin A secretion promotes the immune system and oral health, protecting teeth and mucosa from infections. A larger saliva volume improves the nutritional status by promoting digestion and nutrient availability in the gut $[31,65,71,81]$. Optimizing umami in the food to elderly and hospitalized individuals may well improve their QOL.

Glutamate and umami therefore have a number of attractive effects in relation to improving nutrition and health [44]. Glutamate can be used for dietetic purposes by its ability to encourage an individual's choice of certain foods stimulated by an attraction to umami [2]. Free glutamate in the diet can provoke a visceral response from the stomach, intestines, and portal vein [29]. In this way, glutamate can stimulate the appetite in elderly individuals and hence increase food acceptance and intake. The intelligent uses of umami may provide for better nutritional management and less intake of salt, fat, and sugar without compromising palatability. Umami may also help us to produce flavorful and delicious dishes from only vegetable sources thereby making it easier to meet the recommended daily intake of $600 \mathrm{~g}$ of vegetables and greens.

It is likely that humans' craving for umami goes back to the invention of cooking along with the hominins' use of fire to heat-treat food almost two million years ago. It may also appear that modern humans in the age of industrialization and an individualized food culture have neglected this evolutionary driven undercurrent and "forgotten" that delicious food is not identical to sweet and fatty food and that our species evolved in a food culture where the meals were a uniting and collective endeavor. The current scientific insight into how delicious food with umami is produced and enhanced could potentially be better exploited to optimize and regulate food intake and lead to greater satiety and satisfaction after less comprehensive and less caloric portions [44]. Altogether, a focus on the deliciousness of our food may be a way of counteracting many of the diet-related lifestyle diseases, such as heart and coronary diseases, obesity, diabetes, and hypertension as well as lead to a better quality of life with a stronger social coherence.

\section{Conclusions}

This topical review has argued that our brain and our taste are under siege due to changes in our food and diet, in particular over the last century, leading to massive changes in the burden of ill health for the global population. This situation may best be put into perspective in the context of evolution.

According to conventional Darwinism, there are two principal forces that drive evolution, "natural selection and conditions of existence;" that is, genetic inheritance and the environment [41]. Of the two, Darwin thought that the environment has the greatest influence. This means that even though the genes, and the way in which they are expressed, control the biological function of an organism, the organism is limited by the possibilities afforded by its surroundings and by the laws of physics and chemistry. An important aspect of the environment is food and the manner we prepare it and consume it. In this way, food and food culture enter the picture as a driving force in the evolution of the species [11]. Our genes have only changed little since Homo sapiens came on the scene but the expression of the genes has and does so very quickly.

With this background, one can say that we do not eat the way our genes tell us to eat and this may be a reason for the massive rise in non-communicable, diet-related diseases we have witnessed in the global population over the last century. Heart and coronary diseases, obesity, diabetes, hypertension, cancer, and certain mental diseases are threatening the population, leading to ill health and loss in wellbeing and quality of life.

It is the contention of the present paper that much could be gained by focusing on the fatty acid composition and the deliciousness of our food.

\section{Competing interests \\ The author declares that he has no competing interests.}

\section{Acknowledgments}

This work was carried out while the author held a special center grant from Nordea-fonden to support the Danish National Research and Communication Center Taste for Life. 
Received: 17 March 2016 Accepted: 4 June 2016

Published online: 15 June 2016

\section{References}

1. Araujo IET, Kringelbach ML, Rolls ET, Hobden P. Representation of umami taste in the human brain. J Neurophysiol. 2003;90:313-9.

2. Bellisle F. Experimental studies of food choices and palatability responses in European subjects exposed to the umami taste. Asia Pacific J Clin Nutr. 2008;17(S1):376-9.

3. Bibbins-Domingo K, Chertow GM, Coxson PG, Moran A, Lightwood JM, Pletcher MJ, Goldman L. Projected effect of dietary salt reductions on future cardiovascular disease. N Engl J Med. 2010;362:590-9.

4. Brenna JT, Carlson SE. Docosahexaenoic acid and human brain development. Evidence that a dietary supply is needed for optimal development. J Human Evol. 2014;77:99-106.

5. Brug J, Oenema A. Healthful nutrition promotion in Europe: goals, target populations, and strategies. Patient Educ Couns. 2006;63:255-7.

6. Chen X, Gabitto M, Peng Y, Ryba NJP, Zucker CS. A gustotopic map of taste qualities in the mammalian brain. Science. 2011;333:1262-6.

7. Comody RN, Weintraub GS, Wrangham RW. Energetic consequences of thermal and nonthermal food processing. Proc Natl Acad Sci U S A. 2011; 108:19199-203.

8. Cornish ML, Critchley AT, Mouritsen OG. A role for dietary macroalgae in the amelioration of certain risk factors associated with cardiovascular disease. Phycologia. 2015;54:649-66.

9. Cooper RE, Tye C, Kuntsi J, Vassos E, Asherson P. Omega-3 polyunsaturated fatty acid supplementation and cognition: a systematic review and metaanalysis. J Psychopharmacol. 2015;29:753-63.

10. Crawford MA. A role for lipids as determinants of evolution and hominid brain development. Bio Skr Dan Vid Selsk. 2007:56:7-24.

11. Crawford C, Marsh D. The driving force. Food, evolution, and the future. London: Harper \& Row; 1989

12. Cunnane SC. Survival of the fattest. London: World Scientific; 2005.

13. Cunnane SC. Human brain evolution: a question of solving key nutritional and metabolic constraints on mammalian brain development. In: Cunnane SC, Stewart KM, editors. Human brain evolution: the influence of freshwater and marine food resources. Oxford: Wiley-Blackwell; 2010. p. 33-64.

14. Cunnane SC, Stewart KM, editors. Human brain evolution: the influence of freshwater and marine food resources. Oxford: Wiley-Blackwell; 2010.

15. Cunnane S, Stewart K, Tattersall I. The role of freshwater and marine resources in the evolution of the human diet, brain and behaviour. J Hum Evol. 2014;77:1-216.

16. Drewnowski A. Why do we like fat? J Am Diet Assoc. 1997;97 Suppl 7:S58-62.

17. Dyble M, Salali GD, Chaudhary N, Page A, Smith D, Thompson J, Vinicius L, Mace $R$, Migliano AB. Sex equality can explain the unique social structure of hunter-gatherer bands. Science. 2015;348:796-8.

18. Ebert AG. Response to "Evidence that MSG does not induce obesity.". Obesity. 2010;17:629-30.

19. Ezatti M, Lopez AD, Rodgers A, Hoorn SV, Murray CL, the Comparative Risk Assessment Collaborating Group. Selected major risk factors and global and regional burden of disease. Lancet. 2002;360:1347-60.

20. FAO. The State of World Fisheries and Aquaculture. Rome: FAO; 2012.

21. Gow RV, Hibbell JR. Omega-3 fatty acid and nutrient deficits in adverse neurodevelopment and childhood behaviors. Child Adolesc Psychiatr Clin N Am. 2014;23:555-90

22. Halpern BP. Glutamate and the flavor of foods. J Nutr. 2000;130:910S-4S.

23. Fujii M. The enlightened kitchen. Fresh vegetable dishes from the temples of Japan. Tokyo: Kodanska Int; 2005

24. He FJ, MacGregor GA. Reducing population salt intake worldwide. From evidence to implementation. Prog Cardiovasc Dis. 2010;42:363-82.

25. Hibbeln JR. Omega-3 fatty acid deficiencies and the global burden of psychiatric disorders. Biol Skr Dan Vid Selsk. 2007;56:25-32.

26. Hibbeln JR, Nieminen LR, Blasbalg TL, Riggs JA, Lands WE. Healthy intakes of n-3 and n-6 fatty acids. estimations considering worldwide diversity. Amer J Clin Nutr. 2006:83(Suppl):1483S-93S

27. Hibbeln JR, Davis JM, Steer C, Emmett P, Rogers I, Williams C, Golding J. Maternal seafood consumption in pregnancy and neurodevelopmental outcomes in childhood (ALSPAC study): an observational cohort study. Lancet.

2007;369:578-85
28. Højlund S, Selmer B, Knakkergaard L. Østergaard JS, Dahlgaard V, Bro CP. The flexible boundary between edible and non-edible. Experimental fieldwork in a culinary borderland. Tidsk Antropology. 2014:68:111-129.

29. Jinap S, Hajeb P. Glutamate. Its application in food and contribution to health. Appetite. 2010;55:1-10.

30. Joordensa JCA, Kuipersb RS, Waninkc JH, Muskiete FAJ. A fish is not a fish. Patterns in fatty acid composition of aquatic food may have had implications for hominin evolution. J Hum Evol. 2014;77:107-16.

31. Kawai M, Uneyama H, Miyano H. Taste-active components in foods, with concentration on umami compounds. J Health Sci. 2009;55:667-73.

32. Kondoh T, Mallick HN, Torii K. Activation of the gut-brain axis by dietary glutamate and physiologic significance in energy homeostasis. Amer J Clin Nutr. 2009:90:832S-7S

33. Krebs JR. The gourmet ape: evolution and human food preferences. Am J Clin Nutr. 2009:90:707S-11S.

34. Kromhout D, Menotti $Y$, Kesteloot H, Sans S. Prevention of coronary heart disease by diet and lifestyle. Evidence from prospective cross-cultural, cohort, and intervention studies. Circulation. 2002;105:893-8.

35. Kuller LH. Dietary fat and chronic diseases: epidemiologic overview. J Amer Diet Assoc. 1997;97:S9-S15

36. Levenstein $\mathrm{H}$. Fear of food. A history of why we worry about what we eat. Chicago: University of Chicago Press; 2012.

37. Lévy-Strauss $C$. The raw and the cooked. Mythologiques, volume 1. Chicago: University of Chicago Press; 1983

38. Lieberman DE. The evolution of the human head. Cambridge: Harvard University Press; 2011

39. Lieberman DE. The story of the human body. Evolution, health, and disease. New York: Pantheon; 2013

40. Lucas PW, Ang KY, Sui Z, Agrawal KR, Prinz JF, Dominy NJ. A brief review of the recent evolution of the human mouth in physiological and nutritional contexts. Physiol Behav. 2006;89:36-8.

41. Marsh DE. The origins of diversity. Darwin's conditions and epigenetic variations. Nutr Health. 2007;19:103-32.

42. Miller LR, Jorgensen MJ, Kaplan JR, Seeds MC, Rahbar E, Morgan TM, Welborn A, Chilton SM, Gillis J, Hester A, Rukstalis M, Sergeant S, Chilton FH. Alterations in levels and ratios of n-3 and n-6 polyunsaturated fatty acids in the temporal cortex and liver of vervet monkeys from birth to early adulthood. Physiol Behav. 2016;156:71-8.

43. Mithril C, Dragsted LO, Meyer C, Blauert E, Holt MK, Astrup A. Guidelines for the New Nordic diet. Pub Health Nutr. 2012;17:1-7.

44. Mouritsen OG. Umami flavour as a means to regulate food intake and to improve nutrition and health. Nutr Health. 2012;21:56-75.

45. Mouritsen OG. The emerging science of gastrophysics and its application to the algal cuisine. Flavour. 2012;1:6.

46. Mouritsen OG, Crawford MA. Poly-unsaturated fatty acids, neural function, and mental health. Biol Skr Dan Vid Selsk. 2007;56:1-87.

47. Mouritsen OG, Khandelia H. Molecular mechanism of the allosteric enhancement of the umami taste sensation. FEBS J. 2012;279:3112-20.

48. Mouritsen OG, Williams L, Bjerregaard R, Duelund L. Seaweeds for umami flavor in the New Nordic Cuisine. Flavour. 2012;1:4

49. Mouritsen OG. Seaweeds: edible, available \& sustainable. Chicago: University of Chicago Press; 2013

50. Mouritsen, O. G. (ed.) The science of taste. Flavour 4. 2015. (http://www. flavourjournal.com/series/the_science_of_taste)

51. Mouritsen OG, Styrbæk K. Umami: unlocking the secrets of the fifth taste. New York: Columbia University Press; 2014.

52. Nature Outlook. Making sense of flavour. Nature. 2012;486:S1-S48.

53. O'Donnell M, Mente A, Rangarajan S, for the PURE Investigators, et al. Urinary sodium and potassium excretion, mortality, and cardiovascular events. N Engl J Med. 2014;371:612-23.

54. Olesen J, Gustavsson A, Svensson M, Wittchen $\mathrm{H}-\mathrm{U}$, Jönsson $\mathrm{B}$, on behalf of the CDBE2010 study group and the European Brain Council. The economic cost of brain disorders in Europe. Eur J Neurol. 2012;19: 155-62.

55. Organ C, Nunn CL, Machanda Z, Wrangham RW. Phylogenetic rate shifts in feeding time during the evolution of Homo. Proc Natl Acad Sci U S A. 2011; 108:14555-9.

56. Palou A. Central aspects of sugars in human nutrition. Obes Rev. 2009; 10(Su1):1-58.

57. Peet M, Glen I, Horrobin DF, editors. Phospholipid spectrum disorder in psychiatry. Carnforth: Marius Press; 1999. 
58. Pollan M. The omnivore's dilemma. New York: Penguin Press; 2006.

59. Pomin VM, editor. Seaweed: ecology, nutrient composition, and medicinal uses. New York: Nova; 2011.

60. Prescott J. Taste matters: why we like the food we do. London: Reaktion Books; 2012

61. Reeds PJ, Burrin DG, Stoll B, Jahoor F. Intestinal glutamate metabolism. J Nutr. 2000;130:978S-82S.

62. Rhatigan P. The Irish seaweed kitchen. Co Down, Ireland: Booklink; 2009

63. San Gabriel A, Uneyama $\mathrm{H}$. Amino acid sensing in the gastrointestinal tract. Amino Acids. 2013:45:451-61.

64. Sasano T, Satoh-Kuriwada S, Shoji N, likubo M, Kawai M, Uneyama H, Sakamoto M. Important role of umami taste sensitivity in oral and overall health. Curr Pharm Des. 2014;20:2750-4

65. Schiffman SS. Sensory enhancement of foods for the elderly with monosodium glutamate and flavors. Food Rev Int. 1998;14:321-33.

66. Simopoulos AP. The importance of the ratio of omega-6/omega-3 essential fatty acids. Biomed Pharmacotherapy. 2002;56:365-79.

67. Sørensen LB, Møller P, Flint A, Martens M, Raben A. Effect of sensory perception of foods on appetite and food intake: a review of studies on humans. Int. J. Obes. Relat. Metab. Disord. 2003;27 Suppl 10:1152-1166.

68. Strauss S. Parse the salt, please. Nature Med. 2010;16:841-3.

69. Stender $\mathrm{S}, \mathrm{J}$. Dyerberg \& A. Astrup. High levels of industrially produced trans fat in popular fast foods. N Engl J Med. 2006;354:1650-2.

70. Temussi PA. Sweet, bitter and umami receptors. a complex relationship. Trends Biochem Sci. 2009;34:296-302.

71. Tomoe M, Inoue Y, Sanbe A, Toyama K, Yamamoto S, Komatsu T. Clinical trial of glutamate for the improvement of nutrition and health in the elderly. Ann NY Acad Sci. 2008;1170:82-6.

72. Uneyama H, Kawai M, Sekine-Hayakawa Y, Torii K. Contribution of umami substances in human salivation during meal. J Med Invest. 2009; 56(Supplement):197-204.

73. Vilgis TA. Texture, taste and aroma. multi-scale materials and the gastrophysics of foods. Flavour. 2013:2:12.

74. Weaver KL, Ivester P, Chilton JA, Wilson MD, Pandey P, Chilton FH. The content of favorable and unfavorable polyunsaturated fatty acids found in commonly eaten fish. J Acad Nutr Diet. 2008;108:1178-85.

75. WHO. World Health Organization. Constitution of the World Health Organization-basic documents. 45th ed. Geneva: WHO; 2006.

76. Woschnagg H, Stöllberger C, Finsterer J. Loss of taste is loss of weight. Lancet. 2002;359:891.

77. Wrangham RW. Catching fire: how cooking made us human. New York: Basic Books; 2009

78. Wrangham R, Conklin-Brittain N. Cooking as a biological trait. Comp Biochim Physiol A. 2003;136:35-46.

79. Yamaguchi S. Basic properties of umami and its effects on food flavor. Food Revi Int. 1998:14:139-176.

80. Yamaguchi S, Takahashi C. Interactions of monosodium glutamate and sodium chloride on saltiness and palatability of a clear soup. J Food Sci. 1984:49:82-5.

81. Yamamoto S, Tomoe M, Toyama K, Kawai M, Uneyama H. Can dietary supplementation of monosodium glutamate improve the health of the elderly? Amer J Clin Nutr. 2009;90:844S-9S.

82. Yoshida Y. Umami taste and traditional seasonings. Food Rev Int. 1998;14: 213-46.

83. Zhang FB, Klebansky B, Fine RM, Xu H, Pronin A, Liu H, Tachdjian C, Li X. Molecular mechanism for the umami taste synergism. Proc Natl Acad Sci U S A. 2008:105:20930-4.

84. Zink KD, Lieberman DE. Impact of meat and Lower Paleolitic food processing techniques on chewing in humans. Nature. 2016:532:500-3.

\section{Submit your next manuscript to BioMed Central and we will help you at every step:}

- We accept pre-submission inquiries

- Our selector tool helps you to find the most relevant journal

- We provide round the clock customer support

- Convenient online submission

- Thorough peer review

- Inclusion in PubMed and all major indexing services

- Maximum visibility for your research

Submit your manuscript at www.biomedcentral.com/submit
) Biomed Central 